\title{
MicroRNA-186 targets Yes-associated protein 1 to inhibit Hippo signaling and tumorigenesis in hepatocellular carcinoma
}

\author{
TINGYAN RUAN $^{1}$, XIAOTING HE ${ }^{1}$, JUN YU $^{2}$ and ZHIQIANG HANG ${ }^{1}$ \\ ${ }^{1}$ Department of Oncology, Wuxi People's Hospital Affiliated to Nanjing Medical University, Wuxi, Jiangsu 214023; \\ ${ }^{2}$ Department of Thoracic Surgery, The Second People's Hospital of Wuxi, Wuxi, Jiangsu 214002, P.R. China
}

Received January 15, 2015; Accepted February 16, 2016

DOI: $10.3892 / \mathrm{ol} .2016 .4312$

\begin{abstract}
Liver cancer, particularly hepatocellular carcinoma (HCC), is one of leading causes of cancer-related mortality worldwide. Upregulation of the evolutionary conserved Hippo signaling pathway has been observed in HCC patients, and Yes-associated protein 1 (YAP1) has been reported to play a key role in HCC tumorigenesis. microRNAs (miRNAs) are a family of small non-coding RNAs, usually 21-25 nucleotides in length, and are essential in the regulation of gene expression. Abnormal miRNA expression has been implicated in the initiation and progression of numerous forms of cancers, including liver cancer. Here, we report the identification of a novel miRNA, miR-186, and its functions as an HCC tumor suppressor. We observed that miR-186 was downregulated in several HCC cell lines, and that it directly targets YAP1 mRNA. Overexpression of miR-186 in HCC cells significantly downregulates YAP1 mRNA and protein levels, leading to downregulation of the Hippo signaling pathway, which in turn severely inhibits HCC cell migration, invasion and proliferation. Our study is the first to report the direct involvement of miR-186 in downregulating YAP1 and, more significantly, inhibiting HCC tumorigenesis, and supports the role miR-186 as a potential therapeutic target in treating liver cancer.
\end{abstract}

\section{Introduction}

Human hepatocellular carcinoma (HCC) is one of the most common types of cancer, causing almost 600,000 mortalities annually, worldwide (1). Chronic inflammatory liver disease including viral infection and exposure to chemical carcinogens are the common prologues of HCC. Surgical removal of the tumor and liver transplant are currently the most effective treatment options for HCC, although metastasis

Correspondence to: Dr Zhiqiang Hang, Department of Oncology, Wuxi People's Hospital Affiliated to Nanjing Medical University, 299 Qingyang Road, Wuxi, Jiangsu 214023, P.R. China

E-mail: zqhang1234@163.com

Key words: miR-186, Yes-associated protein 1, Hippo signaling, hepatocellular carcinoma, tumorigenesis and recurrence are extremely common in patients who have undergone resection. Therefore, understanding the molecular mechanisms of HCC tumorigenesis is an essential step in identifying more effective treatments.

The Hippo signaling pathway is an evolutionary conserved cascade, and it was previously reported to play a critical role in liver regeneration and, more significantly, HCC tumorigenesis (2). The machinery of the Hippo signaling pathway consists of Mst1/2, WW45, Lats1/2 and Mob1, and the downstream effectors are Yes-associated protein 1 (YAP1), TAZ and transcriptional enhancer activation domain family members (TEADs) (1). When the upstream effectors are activated they recruit YAP1-TAZ complex, which is transferred into the nucleus to interact with TEADs, and drives target gene expression to promote cell proliferation and suppress apoptosis (3). The disruption of YAP1-TEAD interaction by YAP-like peptides leads to a significant decrease in the tumor growth rate $(4,5)$. The overexpression of YAP1 in transgenic mice was observed to lead to liver outgrowth and HCC (6). YAP1 was reported to be upregulated in over $60 \%$ of HCC patients, and is an independent predictor for patient survival (7). These studies clearly suggest that YAP1 is an essential downstream effector of the Hippo signaling pathway and furthermore a promising drug target for the inhibition of HCC tumorigenesis.

microRNAs (miRNAs or miRs) are a class of 21-25 nucleotide long, small non-coding RNA molecules, which recognize specific complementary sequences predominantly identified in the 3'-untranslated region (UTR) of target mRNAs. miRNAs either repress translation or degrade target mRNAs $(8,9)$. miRNAs have been implicated in various forms of cancer, including liver cancer, by altering the expression of oncogenes or tumor suppressor genes $(10,11)$. One of the most extensively studied microRNAs targeting YAP1 is miR-375. It was initially identified as a negative regulator of YAP1 by targeting its 3'-UTR in HCC patient tissues and human HCC cell lines (12). It was later confirmed that miR-375 also exhibited the same functions in HCC mouse and rat animal models $(13,14)$. Besides miR-375, there have been no other studies on miRNA regulation of YAPl.

In our study, we aimed to identify novel microRNAs regulating YAP1. By in silico analysis we first identified miR-186 as a potential regulator of YAPl mRNA, and observed that miR-186 was downregulated in several human HCC cell lines. 
Further analysis confirmed that YAPl mRNA was a bona fide target of miR-186, and that mRNA and protein levels of YAP1 in HCC cells were downregulated by overexpression of miR-186. Finally, we demonstrated that the induction of miR-186 inhibited migration, invasion and proliferation in HCC cells, suggesting miR-186 as a potential therapeutic target in treating liver cancer.

\section{Materials and methods}

Cell lines. The human normal liver cell lines THLE-2 and THLE-3, and human liver cancer cell lines HepG2, Hep3B and SNU398 were obtained from the American Type Culture Collection (ATCC, Manassas, VA, USA). Cells were cultured according to ATCC instructions and passaged for less than 6 months after receipt for completion of the studies.

miRNA transfection. Has-miR-186 mirVana miRNA mimic was purchased from Applied Biosystems Life Technologies (assay ID MC11753; Pleasanton, CA, USA) along with mirVana miRNA mimic negative control \#1, and transfected into cell lines according to the manufacturer's instructions.

Luciferase reporter assay. The putative miR-186 binding site at the 3'-UTR of YAP1 was cloned downstream of a cytomegalovirus promoter-driven firefly luciferase cassette in a pMIR-REPORT vector (Applied Biosystems). Mutant forms of the luciferase constructs were also generated using standard PCR-based overlap-extension protocols. For luciferase reporter assay, HepG2 cells $\left(3 \times 10^{4}\right)$ were plated in a 24 -well plate and then co-transfected with $400 \mathrm{ng}$ of either has-miR-186 or miR-control (Applied Biosystems), $200 \mathrm{ng}$ of either wild-type or mutant luciferase constructs, and 40 ng of pRL-TK (Promega Corporation, Madison, WI, USA), using Lipofectamine 2000 (Invitrogen Life Technologies, Carlsbad, CA, USA) according to the manufacturer's instruction. Cells were collected $48 \mathrm{~h}$ after transfection and analyzed using the dual-luciferase reporter assay system (Promega Corporation). The pRL-TK vector provided the constitutive expression of Renilla luciferase, and was used as an internal control to correct the differences in transfection and harvest efficiencies. Data are shown as the means \pm standard error of the mean (SEM) of three independent experiments.

$R N A$ extraction and reverse transcription-quantitative polymerase chain reaction $(R T-q P C R)$. Total mRNA was extracted with the mirVANA miRNA isolation kit (Ambion Life Technologies, Carlsbad, CA, USA) according to the manufacturer's instructions. The expression level of miR-186 was quantified using miRNA-specific TaqMan Pri-miRNA assays (assay ID Hs03303293_pri; Applied Biosystems) and normalized by RNU48 control miRNA assay (P/N 001006; Applied Biosystems). Total RNA was purified using the RNeasy MiniPrep kit (Qiagen, Valencia, CA, USA). Total RNA (1 $\mu \mathrm{g})$ was reverse transcribed with the Superscript II First-Strand synthesis kit (Life Technologies) as recommended by the manufacturer. GAPDH mRNA levels were measured for normalization.

Protein isolation and western blot analysis. Protein lysates were extracted using RIPA buffer [50 mM Tris-HCl, pH 7.4, $150 \mathrm{mM} \mathrm{NaCl}, 1 \mathrm{mM}$ EDTA, $1 \%$ Triton $\mathrm{X}-100,1 \%$ sodium deoxycholate, $0.1 \%$ sodium dodecyl sulphate (SDS) and protease inhibitors]. Lysates were centrifuged at 16,000 x g for $10 \mathrm{~min}$ at $4^{\circ} \mathrm{C}$. Proteins were analyzed by SDS-polyacrylamide gel electrophoresis and transferred to nitrocellulose membrane using a semi-dry transfer unit (Bio-Rad Laboratories, Inc., Hercules, CA, USA). The membrane was then immersed in blocking buffer (phosphate-buffered saline and $0.1 \%$ Tween-20) with 5\% nonfat milk for $20 \mathrm{~min}$ and then incubated with rabbit anti-YAP1 (ab52771; Abcam, Cambridge, $\mathrm{UK}$ ) overnight at $4^{\circ} \mathrm{C}$. After washing with blocking buffer, blots were incubated with horseradish peroxidase-conjugated secondary antibody (Life Technologies, Grand Island, NY, USA) for $20 \mathrm{~min}$, washed again with blocking buffer, and visualized using Luminata Forte Western HRP substrate (EMD Millipore, Billerica, MA, USA).

Wound-healing assay. Cells $\left(1 \times 10^{5}\right)$ transfected with mirVana miRNA mimic negative control \#1 and has-miR-186 were seeded into six-well plates following transfection. A linear wound was carefully made using a $100 \mu 1$ sterile pipette tip across the confluent cell monolayer, and the cell debris was removed by washing with phosphate. The migrated distance of the growing edge on the monolayers was measured $24 \mathrm{~h}$ after being wounded.

Cell invasion assay. Cell invasion assay was performed in a 24-well plate with $8 \mu \mathrm{m}$ pore size chamber inserts (BD Biosciences, Franklin Lakes, NJ, USA). Cells $\left(1 \times 10^{5}\right)$ transfected with mirVana miRNA mimic negative control \#1 and has-miR-186 were placed into the upper chamber of each well with Matrigel-coated membrane, which was diluted with serum-free culture medium. The lower compartment was filled with $500 \mu \mathrm{l}$ medium containing $10 \%$ fetal bovine serum as a chemo-attractant. The cells were incubated at $37^{\circ} \mathrm{C}$ in a $5 \%$ $\mathrm{CO}_{2}$ humidified incubator for $24 \mathrm{~h}$. Then cells were exposed to $20 \mu \mathrm{M}$ 5-ethynyl-2'-deoxyuridine for an additional $4 \mathrm{~h}$ at $37^{\circ} \mathrm{C}$. Membrane inserts were removed from the plate and stained using the ENU kit (Invitrogen Life Technologies). The cells were counted in six random microscopic fields for each well, using NIH ImageJ software (http://imagej.nih.gov/ij/).

Cell proliferation and growth assay. Growth and inhibition of growth were assessed by standard MTT assay at time intervals of $24 \mathrm{~h}$. The number of cells was scored as a percentage relative to the mock treatment group. Data are shown as the means \pm SEM of three independent experiments.

Statistical analysis. All data are presented as the means \pm SEM. A two-tailed Student's t-test was used to establish significant differences between groups. $\mathrm{P}<0.05$ was considered to indicate a statistically significant difference.

\section{Results}

miR-186 expression is downregulated in HCC cell lines. Firstly, using the microRNA.org resource, we identified miR-186 as a potential regulator of YAP1 mRNA. As shown in Fig. 1A, miR-186 recognizes two loci in the 3'-UTR of YAPI (NM_006106) at 3286 and 3451. In order to investigate the role of miR-186 in human HCC, we next examined miR-186 
A

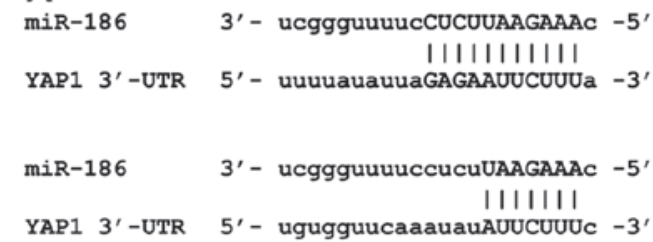

3286

3451

\section{B}

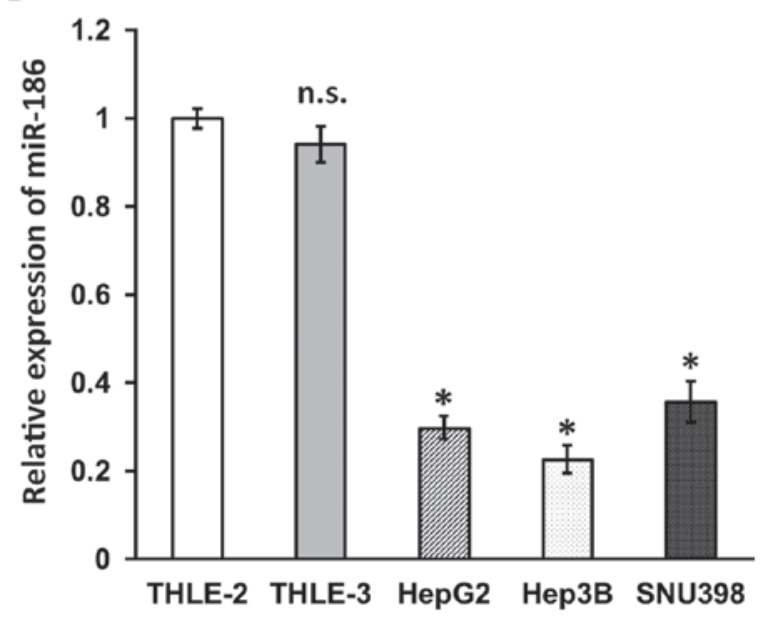

Figure 1. miR-186 expression is downregulated in hepatocellular carcinoma (HCC) cell lines. (A) Sequences of the two putative miR-186 binding sites in the 3'-untranslated region (UTR) of Yes-associated protein 1 (YAP1). Numbers on the right indicate the 3'-UTR position of YAP1 mRNA sequences. (B) Reverse transcription-quantitative polymerase chain reaction analysis of miR-186 levels in two normal human liver cell lines (THLE-2 and THLE-3) and three human HCC cell lines (HepG2, Hep3B and SNU398) relative to THLE-2. RNU48 served as the loading control. Data are shown as the means \pm SEM of three independent experiments. n.s., not significant vs. THLE-2; *P<0.05 vs. THLE-2.

expression in several human HCC cell lines (HepG2, Hep3B and SNU398) and normal human liver cells (THLE-2 and THLE-3) using RT-qPCR (Fig. 1B). miR-186 levels in THLE-2 and THLE-3 were similar. However, miR-186 expression in Hep3B was 30\% lower than that in THLE-2. HepG2 and SNU398 were slightly higher, but still only $30 \%$ and $36 \%$ of the level in THLE-2. These results clearly indicated that compared with normal human liver cells, miR-186 levels were significantly downregulated in human HCC cell lines.

miR-186 directly targets and downregulates YAP1 levels in HCC cell lines. To confirm that YAPl is bona fide target of miR-186, we performed a luciferase reporter assay, using sequences from the original 3'-UTR on YAP1 mRNA as well as three mutated versions (Fig. 2A). MUT1 contained mutations that disrupted only the first potential binding site on YAPI 3'-UTR, while MUT2 mutations affected only the second site, and the MUT1+2 construct contained a combination of both mutated sites. As a result, wild-type luciferase activity was significantly reduced by co-transfection of miR-186, to less than $40 \%$ of miR-control transfected levels, indicating that YAP1 3'-UTR was indeed a direct target of miR-186. Notably, the activity of MUT1 construct was almost $80 \%$ of that of the control, while the activity of MUT2 was reduced to less than $40 \%$ of the control. This suggested that miR-186 targeting to YAPl mRNA was almost entirely through binding to the first
A

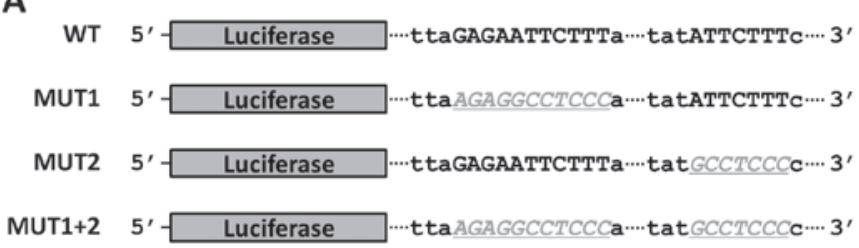

B

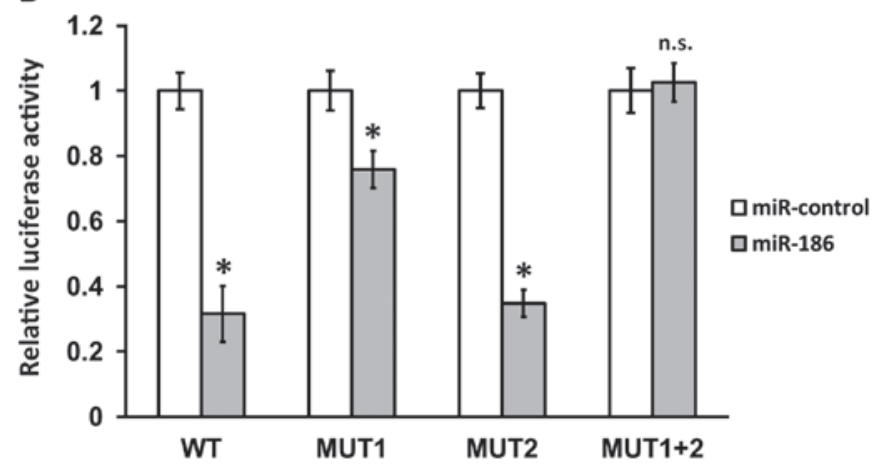

Figure 2. Yes-associated protein 1 (YAP1) is a direct target of miR-186. (A) Schematics of luciferase report construct. Original sequences of two potential binding sites from the 3'-untranslated region (UTR) of YAP1 mRNA were identified in the wild type (WT), and each mutated site is highlighted in MUT1 or MUT2. A combination of both mutated sites was identified in MUT1+2. (B) Luciferase reporter assay in human hepatocellular carcinoma (HCC) cell line HepG2 co-transfected with luciferase construct (WT, MUT1, MUT2 and MUT1+2) and either mirVana miRNA mimic negative control \#1 (miR-control) or has-miR-186 mirVana miRNA mimic (miR-186). Luciferase activity was plotted relative to the respective miR-control. Data are shown as the means \pm SEM of three independent experiments. n.s., not significant vs. respective miR-control; ${ }^{*} \mathrm{P}<0.05$ vs. respective miR-control.

site on the 3'-UTR (Fig. 1A, locus 3286), rather than the second site (Fig. 1A, locus 3451). As expected, the combination of mutations disrupting both binding sites completely blocked the downregulation of luciferase activity of the MUT1+2 construct (Fig. 2B, MUT1+2 n.s.).

We next transfected miR-186 into HCC cell lines and analyzed the mRNA and proteins levels of YAP1. Compared with miR-control transfection, introducing miR-186 significantly reduced the mRNA levels of YAPl in all three HCC cell lines examined (Fig. 3A), suggesting that regulation of YAP1 by miR-186 occurred mainly through mRNA degradation rather than translation repression. Using an antibody against YAP1, we were then able to detect that its protein levels were consistently downregulated in all three miR-186-transfected HCC cell lines, but not in miR-control-transfected cells (Fig. 3B). The expression of connective tissue growth factor (CTGF), a YAP1 downstream effector (3), was also significantly reduced by miR-186 transfection in all three HCC cell lines (Fig. 3C). Taken together, the above results clearly demonstrated that miR-186 downregulated Hippo signaling by directly targeting YAPl mRNA in vivo in HCC cell lines.

miR-186 inhibits proliferation, migration and invasion of HCC cells. As high levels of YAP1 promote metastasis and invasive growth in liver cancer $(1,2,13)$, decreasing YAP1 expression by overexpressing miR-186 in HCC cells should lead to reduced cancer cell proliferation, migration and invasion. To test this hypothesis, we performed an MTT proliferation and 
A

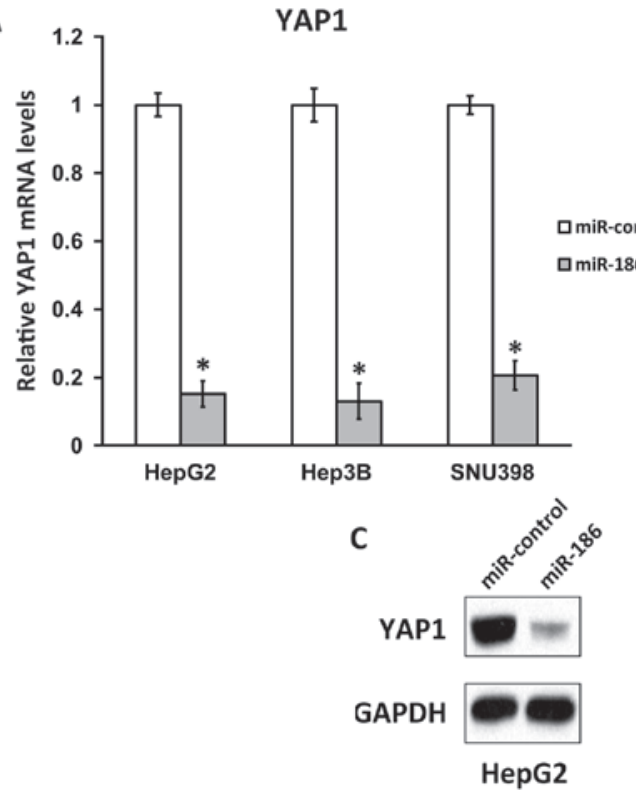

B

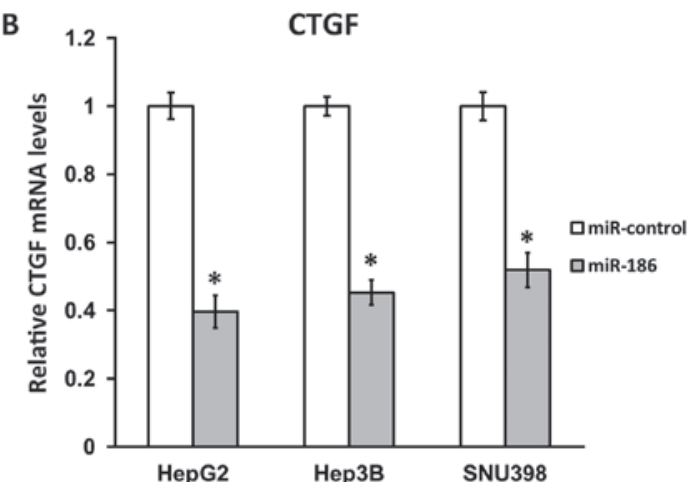

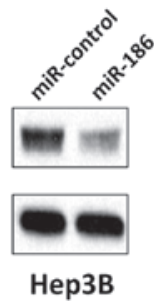

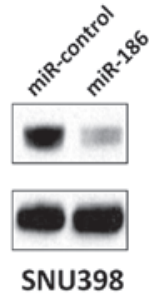

Figure 3. Yes-associated protein 1 (YAP1) level is downregulated by transfection of miR-186 into hepatocellular carcinoma (HCC) cell lines. (A) Reverse transcription-quantitative polymerase chain reaction (RT-qPCR) analysis of YAP1 mRNA levels in three human HCC cell lines (HepG2, Hep3B and SNU398) following transfection with either mirVana miRNA mimic negative control \#1 (miR-control) or has-miR-186 mirVana miRNA mimic (miR-186). YAP1 mRNA levels were normalized to GAPDH, and data are shown as a relative fraction of respective miR-control-transfected cell lines. (B) RT-qPCR analysis of mRNA levels of connective tissue growth factor (CTGF), a downstream target of YAP1, in three human HCC cell lines (HepG2, Hep3B and SNU398). (C) Western blot analysis of YAP1 protein level in three human HCC cell lines (HepG2, Hep3B and SNU398) following transfection with miR-control or miR-186. GAPDH was used as a loading control. Data are shown as the means \pm SEM of three independent experiments. " $\mathrm{P}<0.05$ vs. respective miR-control.

A

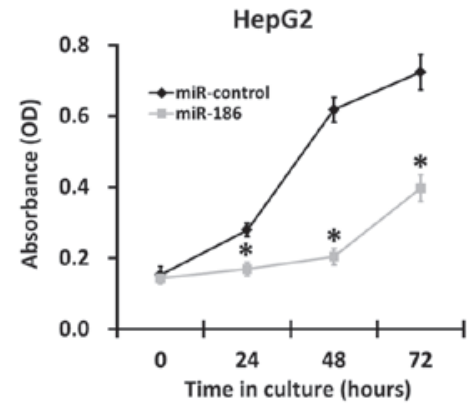

D

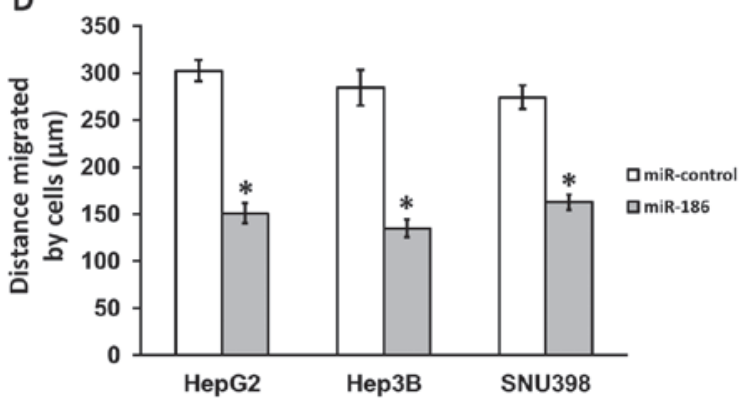

B

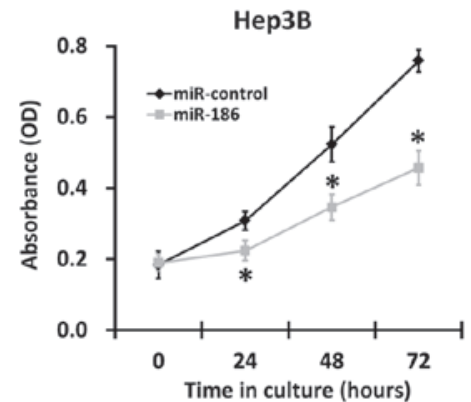

C

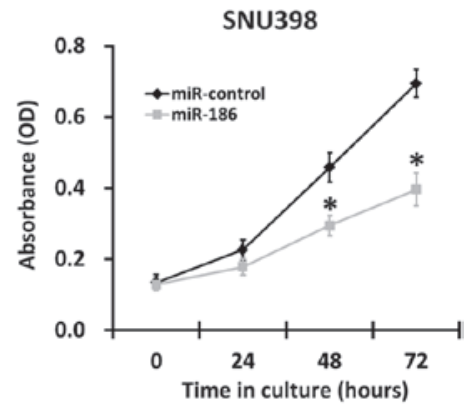

$\mathrm{E}$

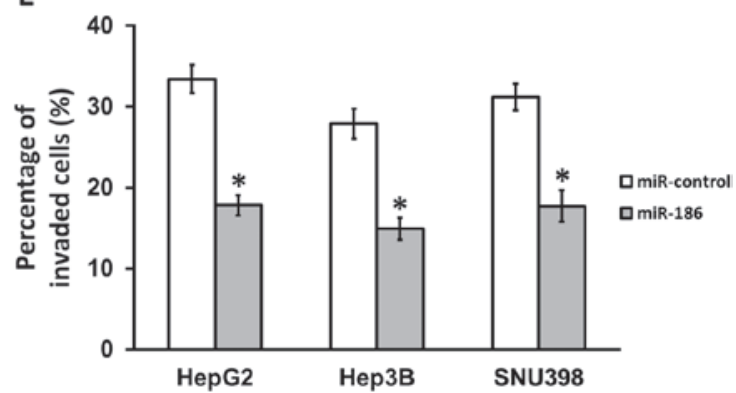

Figure 4. Transfection of miR-186 inhibits hepatocellular carcinoma (HCC) cell migration, invasion and proliferation. (A) Wound-healing assay for three human HCC cell lines (HepG2, Hep3B and SNU398) following transfection with either mirVana miRNA mimic negative control \#1 (miR-control) or has-miR-186 mirVana miRNA mimic (miR-186). The migrated distance of the growing edge on the wounded monolayers was measured $24 \mathrm{~h}$ after being wounded. (B) Cell invasion assay for the three human HCC cell lines following transfection with either miR-control or miR-186. The invaded cell numbers were quantified $24 \mathrm{~h}$ after cells were seeded. (C-E) Cell proliferation and growth assay for HepG2 (C), Hep3B (D) and SNU398 (E) following transfection with either miR-control or miR-186. Data are shown as the means \pm SEM of five independent experiments. ${ }^{*} \mathrm{P}<0.05$ vs. respective miR-control.

growth assay, wound-healing assay and cell invasion assay in miR-186 and miR-control-transfected HCC cell lines (Fig. 4). All three HCC cell lines exhibited a significantly slower growth rate following transfection with miR-186, compared with miR-control experiments (Fig. 4A to C). Moreover, in wound-healing assays, HepG2, Hep3B and SNU398 cells 
transfected with miR-control migrated on average 302, 285 and $274 \mu \mathrm{m}$, respectively, after $24 \mathrm{~h}$. In contrast, when these cells were transfected with miR-186, the distance migrated was notably decreased to 152,135 and $163 \mu \mathrm{m}$ for HepG2, Hep3B and SNU398 cells, demonstrating a reduction of at least $40 \%$ compared with the control (Fig. 4B). As expected, HCC cells transfected with miR-186 also exhibited severe defects in the cell invasion assay compared with those in the control experiments (Fig. 4C). The above results strongly support the role of miR-186 in inhibiting $\mathrm{HCC}$ tumorigenesis.

\section{Discussion}

In the present study, we have identified the Hippo signaling pathway effector YAP1 as being a direct target of miR-186, where two sites on the 3'-UTR of YAP1 mRNA may be recognized by miR-186 with different affinity. miR-186 was usually downregulated in several HCC cell lines we examined, and overexpression of miR-186 in these HCC cells significantly and consistently reduces YAP1 mRNA and protein levels, and in turn downregulates Hippo signaling, which is evidenced by the decreased expression of downstream effector CTGF. Furthermore, introducing miR-186 into HCC cells significantly inhibited their migration, invasion and proliferation.

Various other studies have indicated that miR-186 exhibits multiple functions in a number of aspects of development. It has been noted to downregulate the expression of the pro-apoptotic purinergic $\mathrm{P} 2 \mathrm{x}_{7}$ receptor (15). miR-186 also inhibits muscle cell differentiation through myogenin regulation (16). Plasma miR-186 may be a biomarker for focal segmental glomerulosclerosis with nephrotic proteinuria (17). Moreover, miR-186 surfaced in a previous study as a regulator of acetylcholine packaging and degradation in neuroinflammation-related disorders (18). In a previous study, miR-186 was also implicated as a cancer biomarker. miR-186, together with other miRNAs, was reported to serve as a potential biomarker for oral squamous cell carcinoma (19). Of particular interest, Li et al observed that in a mouse model of human non-small cell lung cancer (NSCLC), miR-186 is able to downregulate pituitary tumor transforming gene PTTG1 and inhibit invasion of NSCLC cells (20). However, there have been no reports of miR-186 involvement in liver cancer.

To conclude, our study has provided the first demonstration that miR-186 functions as a tumor suppressor in HCC cell lines. miR-186 is able to inhibit HCC tumorigenesis by directly targeting YAP1 and downregulating Hippo signaling, which supports its role as a potential application in liver cancer therapy.

\section{Acknowledgments}

This study was supported by the Talented Doctor Funding program (grant no. 20120101).

\section{References}

1. Jie L, Fan W, Weiqi D, Yingqun Z, Ling X, Miao S, Ping C and Chuanyong G: The hippo-yes association protein pathway in liver cancer. Gastroenterol Res Pract 2013: 187070, 2013.

2. Hong L, Cai Y, Jiang M, Zhou D and Chen L: The Hippo signaling pathway in liver regeneration and tumorigenesis. Acta Biochim Biophys Sin (Shanghai) 47: 46-52, 2015.

3. Zhao B, Ye X, Yu J, Li L, Li W, Li S, Yu J, Lin JD, Wang CY, Chinnaiyan AM, et al: TEAD mediates YAP-dependent gene induction and growth control. Genes Dev 22: 1962-1971, 2008.

4. Sudol M, Shields DC and Farooq A: Structures of YAP protein domains reveal promising targets for development of new cancer drugs. Semin Cell Dev Biol 23: 827-833, 2012

5. Zhou Z, Hu T, Xu Z, et al: Targeting Hippo pathway by specific interruption of YAP-TEAD interaction using cyclic YAP-like peptides. FASEB J 29: 724-732, 2015.

6. Dong J, Feldmann G, Huang J, Wu S, Zhang N, Comerford SA, Gayyed MF, Anders RA, Maitra A and Pan D: Elucidation of a universal size-control mechanism in Drosophila and mammals. Cell 130: 1120-1133, 2007.

7. Xu MZ, Yao TJ, Lee NP, Ng IO, Chan YT, Zender L, Lowe SW, Poon RT and Luk JM: Yes-associated protein is an independent prognostic marker in hepatocellular carcinoma. Cancer 115: 4576-4585, 2009.

8. Bartel DP: MicroRNAs: Genomics, biogenesis, mechanism and function. Cell 116: 281-297, 2004.

9. Bartel DP: MicroRNAs: Target recognition and regulatory functions. Cell 136: 215-233, 2009.

10. Volinia S, Calin GA, Liu CG, Ambs S, Cimmino A, Petrocca F, Visone R, Iorio M, Roldo C, Ferracin M, et al: A microRNA expression signature of human solid tumors defines cancer gene targets. Proc Natl Acad Sci USA 103: 2257-2261, 2006.

11. Lu J, Getz G, Miska EA, Alvarez-Saavedra E, Lamb J, Peck D, Sweet-Cordero A, Ebert BL, Mak RH, Ferrando AA, et al: MicroRNA expression profiles classify human cancers. Nature 435: 834-838, 2005.

12. Liu AM, Poon RT and Luk JM: MicroRNA-375 targets Hippo-signaling effector YAP in liver cancer and inhibits tumor properties. Biochem Biophys Res Commun 394: 623-627, 2010.

13. Kowalik MA, Saliba C,Pibiri M,Perra A,Ledda-Columbano GM, Sarotto I, Ghiso E, Giordano S and Columbano A: Yes-associated protein regulation of adaptive liver enlargement and hepatocellular carcinoma development in mice. Hepatology 53: 2086-2096, 2011.

14. Perra A, Kowalik MA, Ghiso E, Ledda-Columbano GM, Di Tommaso L, Angioni MM, Raschioni C, Testore E, Roncalli M, Giordano S and Columbano A: YAP activation is an early event and a potential therapeutic target in liver cancer development. J Hepatol 61: 1088-1096, 2014.

15. Zhou L, Qi X, Potashkin JA, Abdul-Karim FW and Gorodeski GI: MicroRNAs miR-186 and miR-150 down-regulate expression of the pro-apoptotic purinergic $\mathrm{P} 2 \mathrm{X} 7$ receptor by activation of instability sites at the 3'-untranslated region of the gene that decrease steady-state levels of the transcript. J Biol Chem 283: 28274-28286, 2008.

16. Antoniou A, Mastroyiannopoulos NP, Uney JB and Phylactou LA: miR-186 inhibits muscle cell differentiation through myogenin regulation. J Biol Chem 289: 3923-3935, 2014.

17. Zhang C, Zhang W, Chen HM, Liu C, Wu J, Shi S and Liu ZH: Plasma MicroRNA-186 and proteinuria in focal segmental glomerulosclerosis. Am J Kidney Dis 65: 223-232, 2015.

18. Nadorp B and Soreq H: Predicted overlapping microRNA regulators of acetylcholine packaging and degradation in neuroinflammation-related disorders. Front Mol Neurosci 7: 9, 2014.

19. Ries J, Vairaktaris E, Agaimy A, Kintopp R, Baran C, Neukam FW and Nkenke E: miR-186, miR-3651 and miR-494: Potential biomarkers for oral squamous cell carcinoma extracted from whole blood. Oncol Rep 31: 1429-1436, 2014.

20. Li H, Yin C, Zhang B, Sun Y, Shi L, Liu N, Liang S, Lu S, Liu Y, Zhang J, et al: PTTG1 promotes migration and invasion of human non-small cell lung cancer cells and is modulated by miR-186. Carcinogenesis 34: 2145-2155, 2013. 